\title{
Recent Progress on Hadron Structure from Lattice
} QCD

\author{
Jian-Hui Zhang* \\ Institut für Theoretische Physik, Universität Regensburg, D-93040 Regensburg, Germany \\ E-mail: jianhui.zhangeur.de
}

Recent years have witnessed rapid progress on computing Bjorken- $x$ dependence of hadron structure from lattice QCD. In this talk, I give a brief review of such developments, with a particular focus on the isovector quark PDFs in the proton, for which intensive studies in the past few years have yielded very encouraging results.

The 9th International workshop on Chiral Dynamics

17-21 September 2018

Durham, NC, USA

${ }^{*}$ Speaker. 


\section{Introduction}

Since the deep inelastic scattering experiment at SLAC in late 1960s, the hadron structure has been probed in various hard scattering processes. In such processes, the hadron structure can be characterized by certain parton observables such as the parton distribution functions (PDFs) or the distribution amplitudes (DAs). These parton observables are defined as the expectation value of lightcone correlations in the hadron state, and how to calculate them from first principles has been a long-standing challenge in hadron physics. In the past few decades, the ab initio lattice QCD approach has only been able to access the first few moments of the PDFs and DAs [1, 2, 3, 4], while reconstructing the full distribution requires information on all their moments. Phenomenologically, the PDFs or DAs are usually determined by assuming a smoothly parametrized form and fitting the unknown parameters to a large variety of experimental data (for a recent review, see e.g. Ref. [5]).

Recently, a new theory framework has been developed that allows for a lattice calculation of the full $x$-dependence, instead of the moments, of parton observables. This theory is now known as large momentum effective theory (LaMET) [6, 7] (for other related proposals see Refs. [8, 9, $10,11,12,13,14,15,16])$. According to LaMET, a parton observable can be directly accessed from lattice QCD using the following procedure: 1) Construct an appropriate static-operator matrix element (quasi-observable) that approaches the parton observable in the infinite momentum limit of the external hadron. The quasi-observable constructed in this way is usually hadron-momentumdependent but time-independent, and thus can be readily computed on the lattice. 2) Calculate the quasi-observable on the lattice and renormalize it nonperturbatively in an appropriate scheme. 3) Match the renormalized quasi-observable to the parton observable through a factorization formula accurate up to power corrections that are suppressed by the hadron momentum. The existence of such a factorization is ensured by construction. For a proof in the case of nonsinglet quark distribution, see Refs. [8, 9, 17]. In the case of singlet quark distribution and gluon distribution, the proof is given in Ref. [18].

Since LaMET was proposed, much progress has been achieved both in the theoretical understanding of the formalism and in the direct calculation of the $x$-dependent hadron structure from lattice QCD (see a recent review [19] and references therein). In particular, multiplicative renormalization of both the quark [20, 21, 22] and the gluon [23, 24] quasi-PDF has been established in coordinate space. Nonperturbative renormalization in the regularization-independent momentum subtraction (RI/MOM) scheme as well as a perturbative matching in the same scheme has been carried out for the flavor nonsinglet quark quasi-PDFs in Refs. [25, 26, 27, 28] (see also [29, 30, 31]) and for the singlet quark and gluon quasi-PDFs in Ref. [18]. Despite limited volumes and relatively coarse lattice spacings, the state-of-the-art nucleon isovector quark PDFs determined from lattice data at the physical point have shown a reasonable agreement [27, 28, 31] with phenomenological results extracted from the experimental data [32, 33, 34, 35, 36]. Of course, a careful study of theoretical uncertainties and lattice artifacts is still needed to fully establish the reliability of the results.

In this talk, I review recent progress in lattice calculations of the $x$-dependence of hadron structure, with a particular focus on the isovector quark PDFs in the proton, for which intensive studies in the past few years have yielded very encouraging results. 


\section{From quasi-PDF to PDF}

The leading-twist quark PDF in the proton is defined as

$$
q(x, \mu)=\int \frac{d \xi^{-}}{4 \pi} e^{-i x P^{+} \xi^{-}}\left\langle P\left|\bar{\psi}\left(\xi^{-}\right) \Gamma W\left(\xi^{-}, 0\right) \psi(0)\right| P\right\rangle
$$

where the proton has momentum $P^{\mu}=\left(P^{0}, 0,0, P^{z}\right), \psi, \bar{\psi}$ denote the quark fields, $\xi^{ \pm}=(t \pm z) / \sqrt{2}$ are the lightcone coordinates, $x$ is the fraction of proton momentum carried by the quark, $\Gamma=$ $\left\{\gamma^{+}, \gamma^{+} \gamma_{5}, \gamma^{+} \gamma^{\perp} \gamma_{5}\right\}$ is a Dirac structure that specifies the leading-twist quark PDF, $\mu$ is the renormalization scale in the $\overline{\mathrm{MS}}$ scheme, and

$$
W\left(\xi^{-}, 0\right)=\exp \left(-i g \int_{0}^{\xi^{-}} d \eta^{-} A^{+}\left(\eta^{-}\right)\right)
$$

is the Wilson line inserted to ensure gauge invariance of the nonlocal quark correlator, where $A^{+}=$ $A_{a}^{+} t^{a}$ with $t^{a}$ being the generators in the fundamental representation of color $S U$ (3) group.

For the above quark PDF, a well-suited quasi-PDF candidate is given by

$$
\tilde{q}\left(x, \tilde{\mu}, P^{z}\right)=N \int \frac{d z}{4 \pi} e^{i z x P^{z}}\langle P|\bar{\psi}(z) \Gamma W(z, 0) \psi(0)| P\rangle,
$$

where $z$ is a spatial direction, $\Gamma=\left\{\gamma^{t}, \gamma^{z} \gamma_{5}, \gamma^{t} \gamma^{\perp} \gamma_{5}\right\}$ is a Dirac matrix with the corresponding normalization factor $N=P^{z} / P^{t}, \tilde{\mu}$ is the renormalization scale in an appropriate scheme. As shown in Ref. [20], the renormalization of the above quark quasi-PDFs has a multiplicative form in coordinate space so that the matrix elements at different $z$ do not mix with each other. Moreover, the above choice has the advantage of avoiding $\mathscr{O}(1)$ mixing with other PDFs when a non-chiral lattice fermion is used [29, 37].

The renormalization of the above quark quasi-PDF can be carried out nonperturbatively in the RI/MOM scheme [25, 26]. After the bare matrix element on the r.h.s. of Eq. 2.3, denoted as $\tilde{h}\left(z, P^{z}, a\right)$, is calculated on the lattice, it can be renormalized by demanding that the counterterm cancels all the loop contributions for the matrix element of the same operator in an off-shell external quark state at a specific momentum [25, 26]:

$$
\tilde{h}_{R}\left(z, P^{z}, \mu_{R}, p_{z}^{R}\right)=Z^{-1}\left(z, \mu_{R}, p_{z}^{R}, a\right) \tilde{h}\left(z, P^{z}, a\right)
$$

and

$$
Z\left(z, \mu_{R}, p_{z}^{R}, a\right)=\left.\frac{\operatorname{Tr}\left[\Lambda_{\Gamma} \mathscr{P}\right]}{\operatorname{Tr}\left[\Lambda_{\Gamma} \mathscr{P}\right]_{\text {tree }}}\right|_{\substack{p^{2}=-\mu_{R}^{2} \\ p_{z}=p_{z}^{R}}},
$$

where $\Lambda_{\Gamma}$ is the amputated Green's function of the quark bilinear operator in Eq. (2.3) in an offshell quark state with momentum $p . \mu_{R}, p_{z}^{R}$ are renormalization scales introduced in the RI/MOM scheme. $\mathscr{P}$ is a projection operator that defines the renormalization factor [26, 28, 38, 39]. After renormalization, $\tilde{h}_{R}$ has a well-defined continuum limit. It can be converted to the renormalized quasi-PDF, $\tilde{q}_{R}$, by the Fourier transform in Eq. (2.3). When taking the isovector $u-d$ combination, $\tilde{q}_{R}$ can be connected to the normal PDF in the $\overline{\mathrm{MS}}$ scheme through the following factorization 

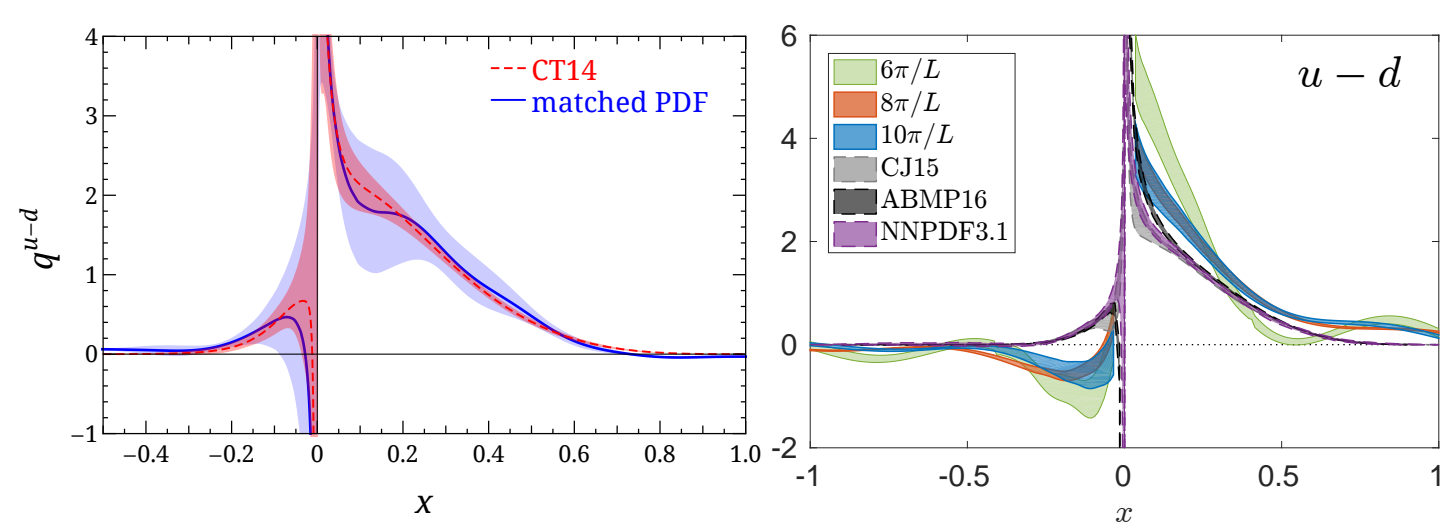

Figure 1: Comparison of unpolarized isovector quark distributions with phenomenological results (left: $\mathrm{LP}^{3}$, right: ETMC).

formula

$$
\tilde{q}_{R}\left(x, P_{z}, \mu_{R}, p_{z}^{R}\right)=\int_{-1}^{1} \frac{d y}{|y|} C\left(\frac{x}{y}, \frac{\mu_{R}}{p_{z}^{R}}, \frac{y P_{z}}{\mu}, \frac{y P_{z}}{p_{z}^{R}}\right) q(y, \mu)+\mathscr{O}\left(\frac{M^{2}}{P_{z}^{2}}, \frac{\Lambda_{\mathrm{QCD}}^{2}}{P_{z}^{2}}\right),
$$

with a perturbatively calculable hard matching kernel $C$. It is worthwhile to point out that the higher-twist contributions shall behave like $1 /\left[x^{2}(1-x) P_{z}^{2}\right]$ instead of $1 / P_{z}^{2}$, as demonstrated in Ref. [40].

\section{Lattice results on PDFs}

Within the LaMET approach, the isovector quark unpolarized, helicity and transversity PDFs have been computed by two different groups: $\operatorname{LP}^{3}[27,28,39]$ and ETMC [31, 41]. In the work of $\mathrm{LP}^{3}$, the calculation was done using clover valence fermions on an ensemble of gauge configurations with lattice spacing $a=0.09 \mathrm{fm}$, box size $L \approx 5.8 \mathrm{fm}$ and pion mass $M_{\pi} \approx 135 \mathrm{MeV}$ with $N_{f}=2+1+1$ (degenerate up/down, strange and charm) flavors of highly improved staggered quarks (HISQ) [42] generated by MILC Collaboration [43]. The gauge links were hypercubic (HYP)-smeared [44] and then clover parameters were tuned to recover the lowest pion mass of the staggered quarks in the sea [45, 46, 47, 48]. Only one step of HYP smearing was used to improve the discretization effects. The calculation of ETMC used gauge configurations generated with the Iwasaki improved gluon action $[49,50]$ and the twisted mass fermion action with clover improvement [51,52]. Their results were obtained with an ensemble of two degenerate light quarks $\left(N_{f}=2\right)$ at maximal twist, with quark masses that were tuned to reproduce approximately the physical pion mass value [53]. The lattice spacing was $a=0.094 \mathrm{fm}$, box size $L \approx 4.5 \mathrm{fm}$ and pion mass $M_{\pi} \approx 130 \mathrm{MeV}$. They applied stout smearing [54] to the links of the Wilson line entering the operator, and tested up to 20 smearing steps and found complete agreement upon renormalization. Both groups have used a momentum smearing [55] that was designed to align the overlap with nucleons of the desired boost momentum, and the largest momentum reached was 3.0 and $1.4 \mathrm{GeV}$, respectively. 

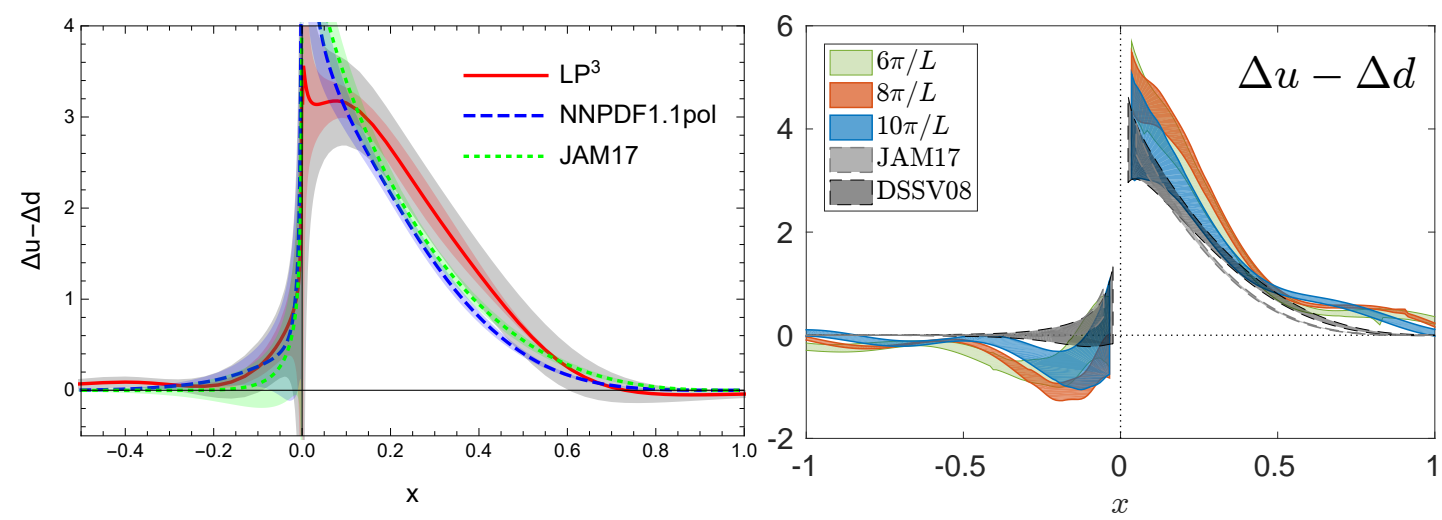

Figure 2: Comparison of isovector quark helicity distributions with phenomenological results (left: LP' right: ETMC).
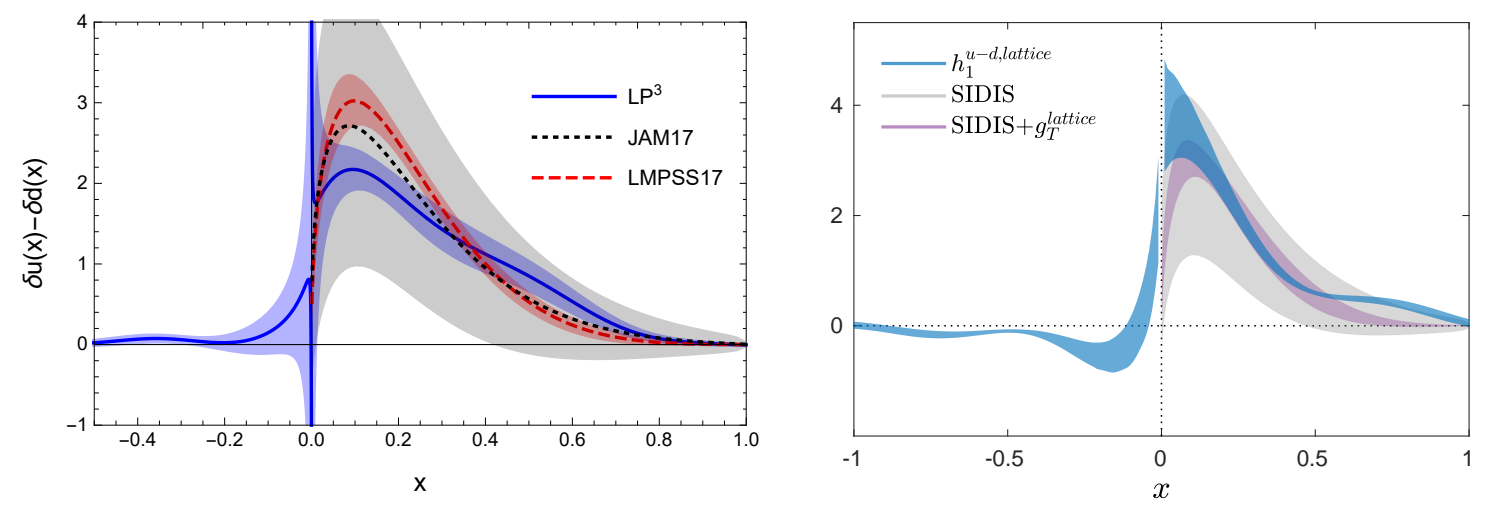

Figure 3: Comparison of isovector quark transversity distributions with phenomenological results (left: LP' right: ETMC).

As can be seen from the ETMC plot in Fig. 1, the lattice results tend to approach the phenomenological curves when the proton momentum is increased. In addition, there is an oscillatory behavior in the ETMC plot, which is due to the truncated Fourier transform from coordinate space matrix elements to momentum space distributions. In the $\mathrm{LP}^{3}$ plot such an oscillation is hardly visible because a "derivative method" [56] has been applied to suppress the unphysical oscillation effects. Similar behavior also occurs in Figs. 2 and 3.

In all three cases, the $\mathrm{LP}^{3}$ results show a reasonable agreeement with phenomenological curves within errors. This is encouraging, however, one should be aware that in these plots a thorough analysis of the systematic uncertainties is still missing, which needs to be done in the future to fully establish the reliability of these results. 


\section{References}

[1] G. Martinelli and C. T. Sachrajda, Pion Structure Functions From Lattice QCD, Phys. Lett. B196 (1987) 184-190.

[2] G. Martinelli and C. T. Sachrajda, The Quark Distribution Amplitude of the Proton: A Lattice Computation of the Lowest Two Moments, Phys. Lett. B217 (1989) 319-324.

[3] W. Detmold, W. Melnitchouk and A. W. Thomas, Parton distributions from lattice QCD, Eur. Phys. J.direct 3 (2001) 13, [hep-lat/0108002].

[4] LHPC, TXL collaboration, D. Dolgov et al., Moments of nucleon light cone quark distributions calculated in full lattice QCD, Phys. Rev. D66 (2002) 034506, [hep-lat/ 0201021 ].

[5] J. Gao, L. Harland-Lang and J. Rojo, The Structure of the Proton in the LHC Precision Era, Phys. Rept. 742 (2018) 1-121, [1709.04922].

[6] X. Ji, Parton Physics on a Euclidean Lattice, Phys. Rev. Lett. 110 (2013) 262002, [1305. 1539 ].

[7] X. Ji, Parton Physics from Large-Momentum Effective Field Theory, Sci. China Phys. Mech. Astron. 57 (2014) 1407-1412, [1404.6680].

[8] Y.-Q. Ma and J.-W. Qiu, Extracting Parton Distribution Functions from Lattice QCD Calculations, 1404.6860 .

[9] Y.-Q. Ma and J.-W. Qiu, Exploring Partonic Structure of Hadrons Using ab initio Lattice QCD Calculations, Phys. Rev. Lett. 120 (2018) 022003, [1709.03018].

[10] A. V. Radyushkin, Quasi-parton distribution functions, momentum distributions, and pseudo-parton distribution functions, Phys. Rev. D96 (2017) 034025, [1705.01488].

[11] K.-F. Liu and S.-J. Dong, Origin of difference between anti-d and anti-u partons in the nucleon, Phys. Rev. Lett. 72 (1994) 1790-1793, [hep-ph/9306299].

[12] J. Liang, K.-F. Liu and Y.-B. Yang, Lattice calculation of hadronic tensor of the nucleon, EPJ Web Conf. 175 (2018) 14014, [1710.11145].

[13] W. Detmold and C. J. D. Lin, Deep-inelastic scattering and the operator product expansion in lattice QCD, Phys. Rev. D73 (2006) 014501, [hep-lat/ 0507007 ].

[14] V. Braun and D. Mueller, Exclusive processes in position space and the pion distribution amplitude, Eur. Phys. J. C55 (2008) 349-361, [0709.1348].

[15] G. S. Bali, V. M. Braun, M. Göckeler, M. Gruber, F. Hutzler, P. Korcyl et al., Pion distribution amplitude from Euclidean correlation functions, in 35th International Symposium on Lattice Field Theory (Lattice 2017) Granada, Spain, June 18-24, 2017, 2017, 1709.04325 , http://inspirehep.net/record/1623222/files/arXiv:1709.04325.pdf.

[16] A. J. Chambers, R. Horsley, Y. Nakamura, H. Perlt, P. E. L. Rakow, G. Schierholz et al., Nucleon Structure Functions from Operator Product Expansion on the Lattice, Phys. Rev. Lett. 118 (2017) 242001, [1703.01153].

[17] T. Izubuchi, X. Ji, L. Jin, I. W. Stewart and Y. Zhao, Factorization Theorem Relating Euclidean and Light-Cone Parton Distributions, 1801.03917.

[18] W. Wang, J.-H. Zhang, S. Zhao and R. Zhu, "A complete matching for quasi-distribution functions in large momentum effective theory.". 
[19] K. Cichy and M. Constantinou, A guide to light-cone PDFs from Lattice QCD: an overview of approaches, techniques and results, 1811.07248.

[20] X. Ji, J.-H. Zhang and Y. Zhao, Renormalization in Large Momentum Effective Theory of Parton Physics, Phys. Rev. Lett. 120 (2018) 112001, [1706.08962].

[21] T. Ishikawa, Y.-Q. Ma, J.-W. Qiu and S. Yoshida, Renormalizability of quasiparton distribution functions, Phys. Rev. D96 (2017) 094019, [1707.03107].

[22] J. Green, K. Jansen and F. Steffens, Nonperturbative renormalization of nonlocal quark bilinears for quasi-PDFs on the lattice using an auxiliary field, Phys. Rev. Lett. 121 (2018) 022004, [1707.07152].

[23] J.-H. Zhang, X. Ji, A. SchÃd'fer, W. Wang and S. Zhao, Renormalization of gluon quasi-PDF in large momentum effective theory, 1808.10824.

[24] Z.-Y. Li, Y.-Q. Ma and J.-W. Qiu, Multiplicative renormalizability of quasi-parton distributions, 1809.01836 .

[25] J.-W. Chen, T. Ishikawa, L. Jin, H.-W. Lin, Y.-B. Yang, J.-H. Zhang et al., Parton distribution function with nonperturbative renormalization from lattice QCD, Phys. Rev. D97 (2018) 014505, [1706.01295].

[26] I. W. Stewart and Y. Zhao, Matching the quasiparton distribution in a momentum subtraction scheme, Phys. Rev. D97 (2018) 054512, [1709.04933].

[27] J.-W. Chen, L. Jin, H.-W. Lin, Y.-S. Liu, Y.-B. Yang, J.-H. Zhang et al., Lattice Calculation of Parton Distribution Function from LaMET at Physical Pion Mass with Large Nucleon Momentum, 1803.04393.

[28] H.-W. Lin, J.-W. Chen, L. Jin, Y.-S. Liu, Y.-B. Yang, J.-H. Zhang et al., Spin-Dependent Parton Distribution Function with Large Momentum at Physical Pion Mass, 1807.07431.

[29] M. Constantinou and H. Panagopoulos, Perturbative renormalization of quasi-parton distribution functions, Phys. Rev. D96 (2017) 054506, [1705.11193].

[30] C. Alexandrou, K. Cichy, M. Constantinou, K. Hadjiyiannakou, K. Jansen, H. Panagopoulos et al., A complete non-perturbative renormalization prescription for quasi-PDFs, Nucl. Phys. $\mathbf{B 9 2 3}$ (2017) 394-415, [1706.00265].

[31] C. Alexandrou, K. Cichy, M. Constantinou, K. Jansen, A. Scapellato and F. Steffens, Reconstruction of light-cone parton distribution functions from lattice QCD simulations at the physical point, 1803.02685 .

[32] S. Dulat, T.-J. Hou, J. Gao, M. Guzzi, J. Huston, P. Nadolsky et al., New parton distribution functions from a global analysis of quantum chromodynamics, Phys. Rev. D93 (2016) 033006, [1506. 07443 ].

[33] NNPDF collaboration, R. D. Ball et al., Parton distributions from high-precision collider data, Eur. Phys. J. C77 (2017) 663, [1706.00428].

[34] L. A. Harland-Lang, A. D. Martin, P. Motylinski and R. S. Thorne, Parton distributions in the LHC era: MMHT 2014 PDFs, Eur. Phys. J. C75 (2015) 204, [1412. 3989].

[35] NNPDF collaboration, E. R. Nocera, R. D. Ball, S. Forte, G. Ridolfi and J. Rojo, A first unbiased global determination of polarized PDFs and their uncertainties, Nucl. Phys. B887 (2014) 276-308, [1406.5539]. 
[36] J. J. Ethier, N. Sato and W. Melnitchouk, First simultaneous extraction of spin-dependent parton distributions and fragmentation functions from a global QCD analysis, Phys. Rev. Lett. 119 (2017) 132001, [1705.05889].

[37] J.-W. Chen, T. Ishikawa, L. Jin, H.-W. Lin, Y.-B. Yang, J.-H. Zhang et al., Operator classification for nonlocal quark bilinear on lattice, 1710.01089.

[38] Y.-S. Liu, J.-W. Chen, L. Jin, H.-W. Lin, Y.-B. Yang, J.-H. Zhang et al., Unpolarized quark distribution from lattice QCD: A systematic analysis of renormalization and matching, 1807.06566.

[39] Y.-S. Liu, J.-W. Chen, L. Jin, R. Li, H.-W. Lin, Y.-B. Yang et al., Nucleon Transversity Distribution at the Physical Pion Mass from Lattice QCD, 1810.05043.

[40] V. M. Braun, A. Vladimirov and J.-H. Zhang, Power corrections and renormalons in parton quasi-distributions, 1810.00048.

[41] C. Alexandrou, K. Cichy, M. Constantinou, K. Jansen, A. Scapellato and F. Steffens, Transversity parton distribution functions from lattice QCD, 1807.00232.

[42] HPQCD, UKQCD collaboration, E. Follana, Q. Mason, C. Davies, K. Hornbostel, G. P. Lepage, J. Shigemitsu et al., Highly improved staggered quarks on the lattice, with applications to charm physics, Phys. Rev. D75 (2007) 054502, [hep-lat/ 0610092 ].

[43] MILC collaboration, A. Bazavov et al., Lattice QCD ensembles with four flavors of highly improved staggered quarks, Phys. Rev. D87 (2013) 054505, [1212 .4768].

[44] A. Hasenfratz and F. Knechtli, Flavor symmetry and the static potential with hypercubic blocking, Phys. Rev. D64 (2001) 034504, [hep-lat/ 010302 9].

[45] R. Gupta, Y.-C. Jang, H.-W. Lin, B. Yoon and T. Bhattacharya, Axial Vector Form Factors of the Nucleon from Lattice QCD, Phys. Rev. D96 (2017) 114503, [1705. 06834 ].

[46] PNDME collaboration, T. Bhattacharya, V. Cirigliano, S. Cohen, R. Gupta, A. Joseph, H.-W. Lin et al., Iso-vector and Iso-scalar Tensor Charges of the Nucleon from Lattice QCD, Phys. Rev. D92 (2015) 094511, [1506.06411].

[47] T. Bhattacharya, V. Cirigliano, R. Gupta, H.-W. Lin and B. Yoon, Neutron Electric Dipole Moment and Tensor Charges from Lattice QCD, Phys. Rev. Lett. 115 (2015) 212002, [1506. 04196 ].

[48] T. Bhattacharya, S. D. Cohen, R. Gupta, A. Joseph, H.-W. Lin and B. Yoon, Nucleon Charges and Electromagnetic Form Factors from 2+1+1-Flavor Lattice QCD, Phys. Rev. D89 (2014) 094502 , [1306.5435].

[49] Y. Iwasaki, Renormalization Group Analysis of Lattice Theories and Improved Lattice Action. II. Four-dimensional non-Abelian SU(N) gauge model, 1111.7054.

[50] A. Abdel-Rehim et al., A first look at maximally twisted mass lattice QCD calculations at the physical point, PoS LATTICE2013 (2014) 264, [1311. 4 522].

[51] R. Frezzotti and G. C. Rossi, Chirally improving Wilson fermions. 1. O(a) improvement, JHEP 08 (2004) 007, [hep-lat/0306014].

[52] B. Sheikholeslami and R. Wohlert, Improved Continuum Limit Lattice Action for QCD with Wilson Fermions, Nucl. Phys. B259 (1985) 572.

[53] ETM collaboration, A. Abdel-Rehim et al., First physics results at the physical pion mass from $N_{f}=2$ Wilson twisted mass fermions at maximal twist, Phys. Rev. D95 (2017) 094515, [1507. 05068 ]. 
[54] C. Morningstar and M. J. Peardon, Analytic smearing of SU(3) link variables in lattice QCD, Phys. Rev. D69 (2004) 054501, [hep-lat/0311018].

[55] G. S. Bali, B. Lang, B. U. Musch and A. Schäfer, Novel quark smearing for hadrons with high momenta in lattice QCD, Phys. Rev. D93 (2016) 094515, [1602 . 05525].

[56] H.-W. Lin, J.-W. Chen, T. Ishikawa and J.-H. Zhang, Improved Parton Distribution Functions at Physical Pion Mass, 1708.05301. 